$r$ DNA is not always properly integrated with the rest of the genome.

\section{Anatomy of Ribosomal DNA}

\section{from our Cell Biology Correspondent}

ON page 454 of this issue of Nature, Birnstiel and his colleagues at Edinburgh-K. Jones and U. E. Loening - describe in detail the properties of the DNA which specifies the structure of ribosomal RNA in the clawed toad Xenopus laevis. A month ago, in two papers in the Journal of Molecular Biology (34, 661 and 681; 1968), Brown and Weber of the Carnegie Institute, working independently, reported virtually identical data. Both groups have exploited the buoyant density centrifugation techniques which Wallace and Birnstiel (1966) developed for separating the ribosomal DNA from the rest of the Xenopus genome, and the RNADNA hybridization methods of Gillespie and Spiegelman.

The essential step in this very elegant work is the clean separation of the ribosomal DNA, and this depends on its high $\mathrm{G} \equiv \mathrm{C}$ content. When the total DNA of Xenopus is centrifuged in a caesium chloride gradient, the ribosomal DNA, denser than the rest, is displaced in the gradient to form a satellite band, which can be collected free from the other fractions. The DNA obtained in this way is double stranded and contains 64 per cent $\mathrm{G} \equiv \mathrm{C}$. It is derived from a single chromosome which contains the nucleolar organizer; and a deletion mutant, which phenotypically lacks the ability to synthesize ribosomes, also lacks this ribosomal DNA.

Both groups agree that the isolated ribosomal DNA contains essentially all the DNA sequences in the Xenopus genome which are complementary to $28 S$ and $18 S$ RNA, and the two genes are present in equimolar amounts. The ribosomal DNA also contains sequences which are not complementary to any of the ribosomal RNAs. According to Brown and Weber, the ribosomal DNA does not, however, contain the sequences for ribosomal $5 S \mathrm{RNA}$; these are not clustered in any DNA fraction but dispersed throughout the entire genome. Quantitative hybridization indicates that there are about 300 to $45028 S$ and $18 S$ RNA genes in the haploid genome, and clustering of ribosomal RNA genes is emerging as a general phenomenon; it has now been found in $E$. coli, B. subtilis and Drosophila as well as Xenopus.

The $28 S$ and $18 S$ RNA genes are very closely linked; so closely that in order to obtain DNA that will hybridize with only one or other of these RNAs, the ribosomal DNA has to be sheared into pieces less than one gene length. Both groups have analysed the arrangement of the ribosomal RNA genes and reached gratifyingly similar results. The most likely arrangement is one consisting of alternating $18 S$ and $28 S$ genes with stretches of DNA, about the same size as the $18 S$ gene, separating each pair. This spacer DNA is not complementary to any ribosomal RNA and its function is unknown, although Birnstiel et al. have shown that it has an unusually high $\mathrm{G} \equiv \mathrm{C}$ content, about 85 per cent.

The picture that thus emerges from the work of both groups is that ribosomal DNA comprises a series of repeating $18 S$ and $28 S$ gene units. This, of course, fits in well with the evidence that, in HeLa cells, ribo- somal RNA is transcribed as a single $45 S$ precursor molecule which is then split to yield $28 \mathrm{~S}$ and $18 \mathrm{~S}$ ribosomal RNA molecules.

\section{Pattern Recognition}

AN interdisciplinary conference bringing together 300 researchers on all aspects of the problems of pattern recognition was held this week at the National Physical Laboratory, Teddington, Middlesex, organized by the Institute of Electrical Engineers. Automated pattern recognition has been getting much publicity lately because of its obvious applications in such areas as postal sorting. A number of research groups at the GPO reported work on the problems of character recognition, a particularly difficult problem when considering hand-written characters, but a group at the GPO's Dollis Hill Research Station plans to have a laboratory model capable of reading either typewriting or hand-written block capitals within 3 years. The Post Office problem is simpler than many other practical applications, however, for the machine's reject rate can be quite high as long as all those letters accepted are read correctly; those rejected are then sorted by hand.

The problem of all such visual recognition systems is nearly the same, though the methods of solution and degree of accuracy may vary - to find the minimum number of relevant features needed by a training set to identify a character. A number of systems examine the behaviour and placement of characters on a matrix of cells, and by a combination of smoothing-getting rid of kinks and discontinuities in the way the figure is written-and thinning-reducing the figure to its essential lines-the character can be classified into one of the determined sets.

What makes this work even more interesting is the close correlation to neurophysiological theory of vision and brain function. A group at the Massachusetts Institute of Technology is attempting to build a computer that can describe and make decisions about an environment. The immediate purpose of this machine is to place a television camera on Mars in the $1970 \mathrm{~s}$ which is capable of looking around, focusing and deciding what information is important enough to transmit back to Earth. To do this the group is constructing a computer program that performs the same functions as the cells in the core of the reticular formation; information input is compared with recorded images in the computer, and if further investigation is thought necessary the organism moves to a second location where the object is again compared to the internally generated image. By building up a stereoscopic picture of the environment using two lenses and a television camera connected to a computer, the system operates in a fashion similar to the retina of the eye. These inputs are then fed into a series of computing modules similar to the reticular formation, each of which receives a random sample of inputs, guesses at their relative importance and is informed of the random samples of the other modules. These preliminary guesses are circulated several times before a decision is reached. Just what decisions the system will be able to make, especially when faced with a totally new environment, remain to be seen, but a computer that can think for itself should have more immediate applications, at least so the MIT group hopes. 